\title{
Development of task-oriented ROS-based Autonomous UGV with 3D Object Detection
}

\author{
Rajesh Raveendran $^{1 *}$, Siva Ariram ${ }^{1 *}$, Antti Tikanmäki ${ }^{1}$ and Juha Röning ${ }^{1}$
}

\begin{abstract}
In a scenario where fire accidents takes place the priority is always human safety and acting swiftly to contain the fire from further spreading. The modern autonomous systems can promise both human safety and can perform actions rapidly. One such scenario which is motivated by urban firefighting was designed in challenge 3 of MBZIRC 2020 competition. In this challenge the UAV's and UGV collaborate autonomously to detect the fire and quench the flames with water. So, in this project we have developed Robot Operating System (ROS)-based autonomous system to solve the challenge for UGV criteria by detecting targeted objects in real-time, in our case its simulated fire and red colored softballs. Then finally localize those targets as markers in the map and navigate autonomously to all those targets. This work has two sections, in the first section mapping and localizing the fire and softballs in highly cluttered environment and then reaching those targets autonomously. Robustly mapping the area with adequate sensors and detection of targets with optimally trained CNN based network is the key to localizing of the targeted objects in a highly cluttered environments.
\end{abstract}

Keywords: ROS, architecture, mapping, autonomous navigation, object detection, $\mathrm{CNN}$, dataset

\section{INTRODUCTION}

Autonomous mobile systems have received more attention in recent days and slowly is integrating into daily activities of human life. Especially in disaster prone situation these systems can be deployed rapidly and contain the disaster. Recently in China, autonomous mobile robots were used to provide medicines to COVID-19 affected patients. Autonomous drones were used to monitor the people crowd and announcing awareness regarding COVID-19 in densely populated countries such as India and China, also in UAE. The unprecedented situation can be further contained if we have online shopping where last mile robots can deliver daily needs to our door steps, this can avoid further human contacts. In order to deploy autonomous systems in these scenarios we need very robust autonomous systems which can navigate in any cluttered environments safely and robustly. In robotics world there are many competitions that are conducted around the world to enhance the overall system robustness and accuracy of autonomous systems and they have to collaborate with each other. One such event is MBZIRC 2020 Challenge[5] which contains three challenges, collaborative UAVs to grab softballs with $11 \mathrm{~cm}$ in diameter in highly dynamic environment, collaborative UAVs and UGV

\footnotetext{
${ }^{1}$ Biomimetics and Intelligent Systems Group(BISG), University of Oulu, 90570 Oulu, Finland. rajesh.raveendran@oulu.fi siva.arirameoulu.fi

* Rajesh Raveendran and Siva Ariram have equally contributed to this work as first authors

FSB Dataset - https://github.com/sivaariram/Fire-and-Ball-Dataset
}

for construction and finally urban firefighting scenario. In this work we have focused on the UGV's criteria for third scenario to localize simulated fire and navigate autonomously in a highly cluttered environment.The designed autonomous navigation system includes real-time path planning, mapping, obstacle avoidance and default recovery phase. We trained a Convolution Neural Network with training data-set using Darknet detection algorithm. Achieving the accuracy in object detection with data-set from different scenarios and training the network for highly optimized weights was one of the most challenging part.

\section{BACKGROUND}

In the development of any Simultaneous Localization and Mapping (SLAM) based autonomously navigating mobile systems, deciding the choice of representation of the environment is a critical design decision. Fundamentally, the environment can be represented in two ways based on features in the environment and dense representation such as occupancy grid ma. And based on the choices SLAM's can be classified into two approaches, namely grid-based and feature-based approaches. In the feature-based approach, the features contain both semantic and metric information. It provides semantic features such as point, line and plane and metric features such as range and orientation [6]. And based on these features, a feature map is incrementally built and using this feature map the mobile system is simultaneously localized in the environment. Feature based maps are highly preferred in structured environment with open space with predefined landmarks [6] [7] and this also depends on what type of features some line based feature extractors provide optimum results in indoor with concrete wall and pillars. And feature-based approach is computationally less expensive compared to dense grid-based approach. Typical indoor environment using Rao-Blackwellized particle filters (RBPFs) are a high-performance solution, and has been integrated into open source software such as Gmapping [7] [8]. The data association step can fail if there are uncertainties arising from the scan data which in turn can lead to incorrect feature extraction [9] [10]. Hence, accuracy of scan data plays a vital role in feature extraction and grid mapping. Also, any type of sensor uncertainties has a probability to propagate into mapping and navigation stack. In this work we have chosen Hector Map [11] and OctoMap [12] which is chosen based on the base controller mode of operation. Most of the localization algorithms utilizes the extended Kalman filter (EKF), unscented Kalman filter (UKF) or Adaptive Monte-Carlo Localization (AMCL) par- 
ticle filters. The Kalman Filter is based on Bayesian method and it computes recursively the optimal parameter estimation from its posterior density. The object dynamic function and posterior density are Gaussian distribution in Kalman filter and the process and measurement functions are linear. In EKF, the process function linearization is performed with Taylor series approximation and in UKF the approximation is based on sigma point sampling. In this project cascading EKF with different modalities has been implemented using robot_localization [14] package.

In order to achieve the high accuracy in object detection at different scales, the recent studies applied multiple layer architecture. At the same time the low-level feature in superficial layers may not perform the detection with high accuracy [4]. Most of the object detection algorithm is totally depend on training data-set and training parameters which helps to prevent the network over trained or under trained. Hence, we decided to collect enough data-sets under different scenarios and depends on number of classes we configured the training parameters. The other important factor is that the detection should not affect the system performance since most of the detection network occupies the GPU memory[1], so we implemented the network based on system performance.

\section{SYSTEM DESCRIPTION}

The UGV platform, Mörri, is an evolutionary version of the high-performance platform that was first used ten years ago in Elrob 2008. Since then, the platform has undergone several development cycles, especially on sensors and computers, but the core features of powerful $5 \mathrm{~kW}$ motors, narrow body and large footprint relative to weight has remained the same. Figure 1 shows the sensors and cameras attached to the Mörri.

Mörri is equipped with custom made battery modules. Each module has $5 \mathrm{Ah} 24 \mathrm{~V}$ capacity with integrated monitor and charger. Robust CAN bus is used to control each battery module (on/off, charging, etc.) and to read its status (how much charge left, temperature etc.). Operation time of Mörri per one battery module is highly depending on the mass of payload, demanded speeds and accelerations, and environmental factors such as ground softness and slope. As a rough approximation, with one battery module Mörri can travel $2 \mathrm{~km}$ on hard surface. The number of batteries can be tuned according to each scenario's parameters so that "enough" batteries are attached to Mörri. Currently, Mörri has space for up to 12 battery modules. Mörri has internal onboard computer Zotac with NVIDIA Geforce 1070 GPU enabling high performance CUDA processing. The complete navigation stack with obstacle avoidance and 3D model reconstruction are performed using this on-board computer so Mörri can operate independently without external connection. Two different options for inter-process communication are available, the commonly used ROS interfaces and a proprietary high performance binary protocol tailored for real time control. Each ROS node utilizes messages from common_msgs stack in-order to avoid circular dependencies

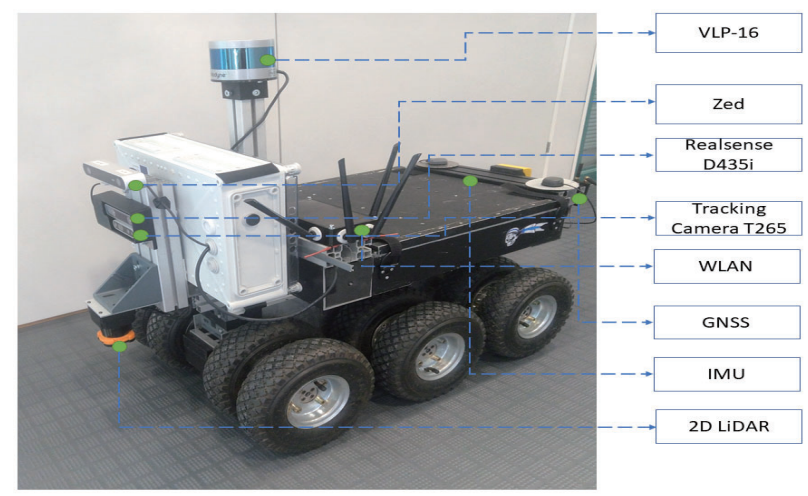

Fig. 1: Mörri UGV

with multiple stack. The system network is configured to use multiple machine with single ROS master. The Operator PC can be used to monitor the heartbeat of all the sensors and receive the messages.

\section{SYSTEM ARCHITECTURE}

Mörri runs in meta-operating system called ROS. The main reasons for opting ROS is easy construction of distributed computing systems, extensive tool-sets for debugging, visualization, logging, introspecting and saving the data in a bag format, has capability to provides libraries from low level system integration to control and perception of mobile systems and manipulators, it is supported by large ecosystem and improved constantly. Figure 2 depicts the software architecture developed based on ROS middleware.

The Mörri's software architecture was developed with several open-sourced packages in Kinetic distro and packages that aid the integration of the available packages were developed. Also, some of the packages source codes were modified according to the system's specifics and computational efficiency. Most of the salient packages are built from source to improve the performance. Table I lists the packages used to construct the software architecture of Mörri.

TABLE I: ROS packages for specific components

\begin{tabular}{|c|c|}
\hline Components & Packages \\
\hline Emlid Reach & reach_rs_ros_driver \\
\hline Intel D435i & Intel RealSense SDK 2.0, realsense-ros \\
\hline Hokuyo UTM 30LX (2D) & hokuyo_node \\
\hline VLP-16 Puck LITE & vsens_driver \\
\hline Xsens MTi-G & mavros \\
\hline Pixfalcon PX4 & hector_slam \\
\hline Hector Map & octomap \\
\hline OctoMap & robot_localization \\
\hline Localization & ros_planning/navigation, teb_local_planner \\
\hline Navigation Stack & joy_stick_drivers \\
\hline F710 Joystick & rviz,MapViz \\
\hline Visualization & darknet_ros_3d \\
\hline Darknet &
\end{tabular}

\section{DATA-SET COLLECTION \& PREPARATION}

The most important fundamental of object detection is collecting data-set, the different scenarios is the key attribute 


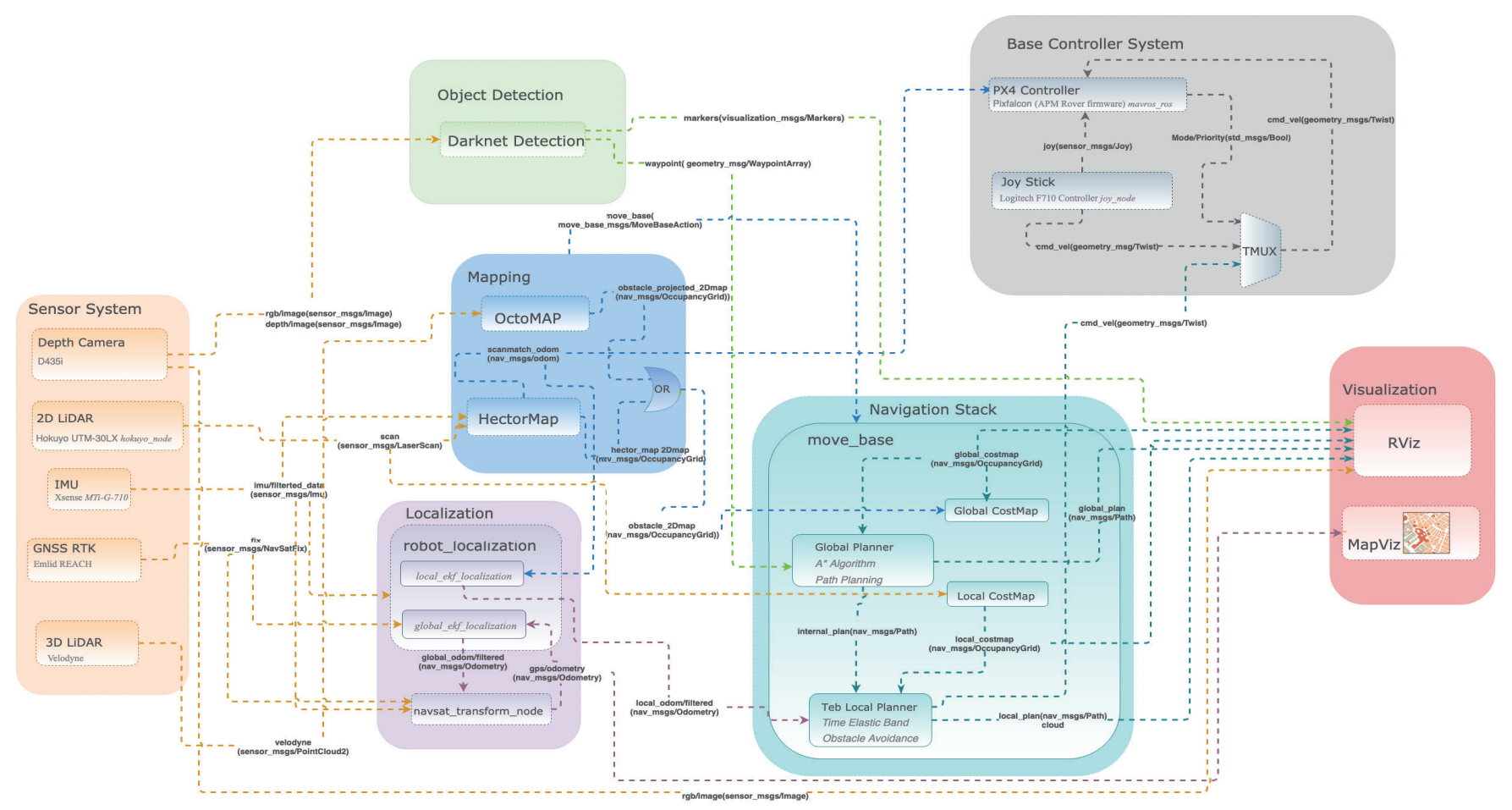

Fig. 2: ROS-based software architecture

of data-set which provides multi angle view of the target. This improves the accuracy of detection under any circumstances. The data-set's original images were captured in the University of Oulu warehouse in summer 2019, it has variety of viewpoints. To support in the reproduciblity of image classification and target recognition we have collected, labelled, generated the images in the Fire simulator and ball(FSB) data-set. Considering the results we achieved when training Darknet using FSB data-set, we note that it provides high quality and various images that are shown to be sufficient to train. For the data collection two different aerial robot vehicle and one ground robot vehicle were used.
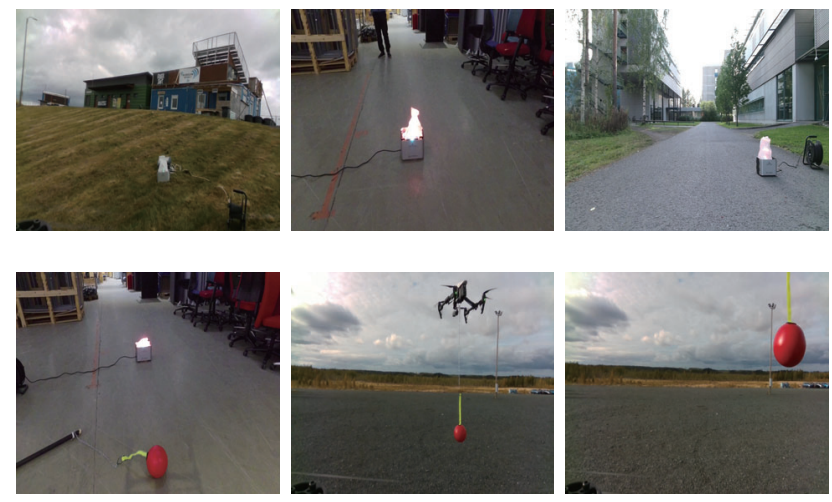

Fig. 3: Example images of indoor and outdoor data-set from both UGV \& UAV

In this compilation, the outdoor data-set of 67276 images and indoor data-set of 33600 images were collected. Im- ages were captured using Realsense D435i Stereo camera, DJI Inspire Camera and Zed Stereo Camera. The sensors mounted in UAV and UGV vehicle's and yield high dynamicrange(HDR) images with 16bits linear color depth. We also included some of low dynamic-range(LDR) images with 8bit RGB images for comparability and compatibility of the data-set[3]. The labelling annotation was done for randomly chosen 1496 frames in a video snippet of different sensors. Figure 3 shows the randomly chosen images from the dataset and it also shows that data-set has variety of viewpoints under different scenarios. The following components of the training/validation sets have to be the images with annotated objects and the text file corresponding to each image which includes the following parameters,

- Label_ID - Numeric ID which has to be starting from 0 , determines different classes.

- X_CENTER - Centre of the object lies on X coordinate

- Y_CENTER - Centre of the object lies on Y coordinate

- WIDTH - Width of the object

- HEIGHT - Height of the object

YOLO Mark is the tool which is used for producing such labels and text files.

\section{METHODOLOGY}

In this section the fundamental components of the software architecture are explained in detail along with the training of Mörri network.

\section{A. Base Controller System}

The base controller is Pixfalcon PX4 with latest Rover firmware version stable-3.6. The Mörri platform can be op- 
erated in three different modes manual, manual with collision avoidance and autonomous with obstacle avoidance, the corresponding modes in PX4 are MANUAL which allows RC, MANUAL with RC override which allows joystick drive and GUIDED. The PX4 controller uses vision based position and orientation rather than its internal filtered IMU which is not suitable in indoor as heading has sporadic errors. The PX4 uses odometry from the 2D LiDAR scan match when $\mathrm{RC}$ messages are overrided by joy stick during manual with collision avoidance. And in the case of GUIDED mode the odometry is similar to the odometry used in local planner or SLAM which is either based on OctoMap or Hector map selection. In upcoming Mapping section this is explained with further details.

\section{B. Localization}

One of the essential methods for position estimation of an autonomous system is odometry/dead reckoning. In ROS, odometry message comprises of position, orientation and twist (linear and angular velocity). The message represents the transformation of the base link frame of the mobile system viewed from the odometry frame. The odometry message is published in odometry frame (odom) as the message itself the transformation of the base link observed from odometry frame. However, the odometry has gradual error accumulation when the robot moves over the time. This gradual drift in odometry is compensated as a transform message from map to odometry frame and most of the SLAM techniques based on feature or grid-based will provide this transform.

In this architecture the state estimation of the UGV is based on cascading the odometry locally and globally from different modalities. The scan-match odometry from 2D LiDAR scan data at $40 \mathrm{~Hz}$ and filtered IMU data from xsens is fused with local_ekf_localization node which provides robust local state estimation of UGV at local frame. Finally this odometry message is used by local planner in navigation stack. The navsat_transform_node provides odometry message which takes heading of the UGV from IMU and fused odometry message from global_ekf_localization node. It finally provides odometry message which is cascaded with global_ekf_localization along with GNSS and odometry message from local_ekf_localization node. The resultant state estimation from global_ekf_localization node is published in consistent with UGV's world frame is highly accurate state of the UGV in world frame.

\section{Mapping}

SLAM is a technique used to localize the mobile system and generate a physical map of the environment simultaneously. And this is always considered to be a complex problem because for mapping the environment a good estimate of the robot's location is needed and to localize a robot, a consistent map of the environment is required. Hence, there is mutual dependency between the estimates about the pose of the robot and the map of the environment. As the task does not require dense model of the environment, we have adopted occupancy grid method rather dense 3D SLAM approaches. In this architecture there are two mapping capabilities. The OctoMaps were used to generate 3D occupancy grid which is more computationally efficient to generate rather graph based RGBD-SLAM such RTAB-Map which is GPU intensive and computationally its not efficient for the given task and storage consumes more space. The OctoMaps are generated from the 3D scan data from velodyne and the projected grid map is used for global and local path planning.

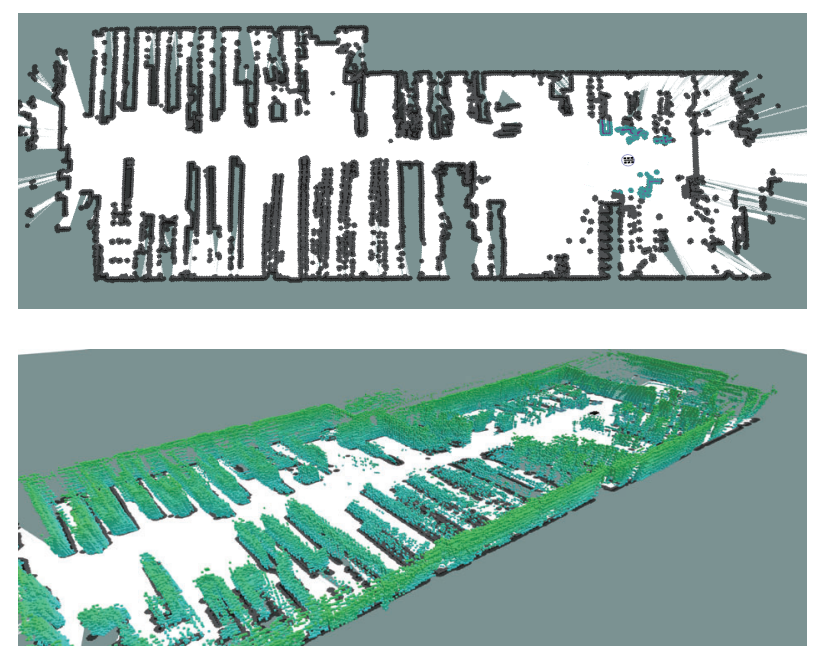

Fig. 4: 2D Hector Map(Top) and 3D OctoMap(Bottom) of the warehouse

In addition to this graph based 2D LiDAR Hector map is used by local planner to avoid obstacles more computationally effective and faster when the global planner is carrot planner, this is used when the base controller in MANUAL mode and overrides by joystick. The base link frame of UGV is stabilised with IMU and it uses scan match odometry generated from 2D LiDAR scans, no EKF fusion is performed from other modalities for Hector SLAM. This promises faster convergence of estimation for mapping compared to EKF based SLAM. As the frequency of the 2D LiDAR is $40 \mathrm{~Hz}$ the system can generate robust map even in long corridors more accurately in large warehouse with corridors larger than 15 meters. The Figure 4 depicts both 2D and 3D maps generated by Hector Map and OctoMap. Also the hector map testing in GUIDED mode boosted the performance of local planner for the navigation because of significant reduction in CPU load with same controller and planner frequencies. Overall, both the mapping has different advantages from convergence of local planner, computation and projection of 3D obstacles stand point.

\section{Navigation}

This is the most crucial part of the overall architecture of Mörri, UGV. Time Elastic Band (TEB) [13] local planner is used for dynamic obstacle avoidance in Mörri. TEB provide multiple path with different topologies which are time optimal solutions. However, TEB solves for unconstrained 
optimization problem which is much faster than constrained optimization problems, the performance of TEB is based on size of the map, size of the obstacle (polygon or circle) and computational power. In this system the parameters are tuned for optimal results considering the computation power with maximum CPU load of other packages and achieving maximum speed for completing the task faster. The maximum velocity achieved was $0.7 \mathrm{~m} / \mathrm{sec}-1.2 \mathrm{~m} / \mathrm{sec}$ along $\mathrm{x}$-axis without compensating the performance of the controller's stability. Figure 8 show the local and global path generated by planners in different scenarios. Navigating in tight corners is much easier with circular footprint as most of the unconstrained problem is now convex optimization problem with smooth costmaps.

\section{E. Object Detection}

To perform object detection, we use the data-sets that provides information about the fire simulator through the camera. The objects are detected using the information from the Realsense D435i camera, then they are classified and the distance of the object relative to the Mörri is measured. The object detection is attained using the integration of feature based and appearance based modeling. As compared to other sensors the images from the camera have more information that can be used to identify objects.

There are plenty of convolutional neural network(CNN) architectures that performs object detection method using feature extractor which are performed better than other methods, this shows development in object detection. During the training process CNN learns the features in object and it can also generalize the object feature.Here, we used existing method which is Darknet object detection and it uses a hierarchical dimension of object classification[1]. Using this method we trained Mörri Object detection network that can detect targeted objects in real-time. Figure 5 shows the realtime detection from Mörri view with the bounding boxes. To find the depth of the object we used depth frame in realsense D435i, we overlapped the bounding box parameters from RGB frame to depth frame to find the depth parameter of the targeted object.
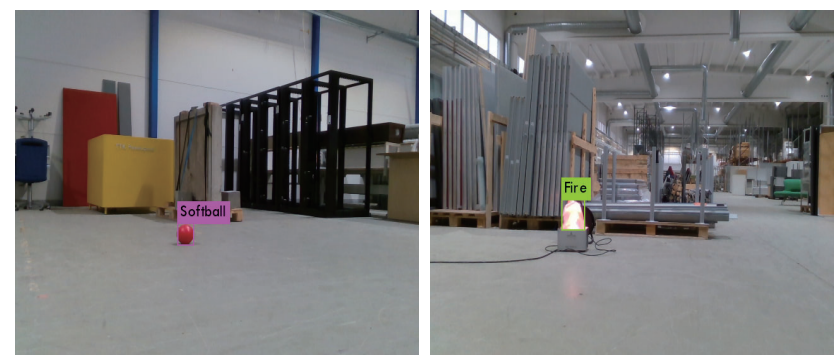

Fig. 5: Trained Mörri network can detect fire simulator and soft ball(Mörri Camera View)

\section{F. Training Network}

To analyse the quality and other attributes of FSB dataset, we utilized widely used open source neural network framework Darknet. The darknet model is trained with high resolution images and shown promising results in object recognition. Figure 6 shows the average training loss of 0.1351 for 4000 iterations on Mörri network which got trained using FSB data-set.

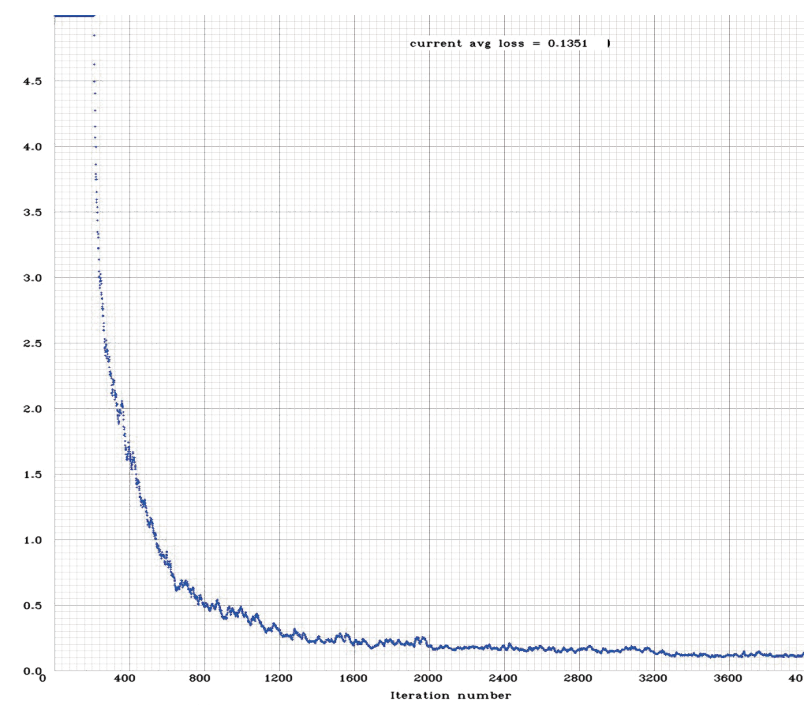

Fig. 6: Plot for average training loss of 4000 iterations

Again under training the model we developed networks with different training regimes, parameters or data-sets, which can also be train depends on system architecture. The Mörri network consists of 19 convolutional layers which got modified with the extraction to be faster with high accuracy. The other parameters for training such as batch size, subdivisions, iterations, etc., are decided based on the number of classes i.e 2 in this task. However, performance rises significantly as the IOU threshold decreases indicating promising results to get the boxes perfectly aligned with the object[1].

\section{EXPERIMENTAL RESULTS}

All the network is trained with the identical parameters and tested at 512 x 512, the Mörri network which is suitable for high end system architecture and it gives mean average precision of $90.89 \%$ with $\sim 32$ FPS, on the other hand it might affect the processing speed of low level configuration system, because it has plenty of layers in its network, Table II shows the mean average precision of three different networks.To overcome the processing speed issue we developed some other networks which have less number of layers and its very precise for low level architecture with mAP of $86.16 \%$ with $\sim 25$ FPS. During the test we experimented the object detection under different scenarios and distance, we could see promising results under different lighting conditions with distance of $\sim 6-7$ metres. The visualization of $3 \mathrm{D}$ bounding boxes were perfectly aligned with the map frame in proper time synchronization[15]. Figure 7 illustrates the real world implementation for Mörri's autonomous navigation, it navigates in the local path generated by TEB planner in GUIDED mode by generating way-points of the target in real-time. 
TABLE II: Three Networks trained for different system architecture

\begin{tabular}{|c|c|c|c|}
\hline Network & Object & Average Precision & mAP \\
\hline \multirow{2}{*}{ Mörri } & Fire Simulator & $90.91 \%$ & \multirow{2}{*}{$90.89 \%$} \\
& Soft Ball & $90.87 \%$ & \\
\hline \multirow{2}{*}{ Copter } & Fire Simulator & $84.72 \%$ & $86.16 \%$ \\
& Soft Ball & $87.59 \%$ & \\
\hline \multirow{2}{*}{ Tiny } & Fire Simulator & $84.79 \%$ & $85.76 \%$ \\
& Soft Ball & $86.73 \%$ & \\
\hline
\end{tabular}
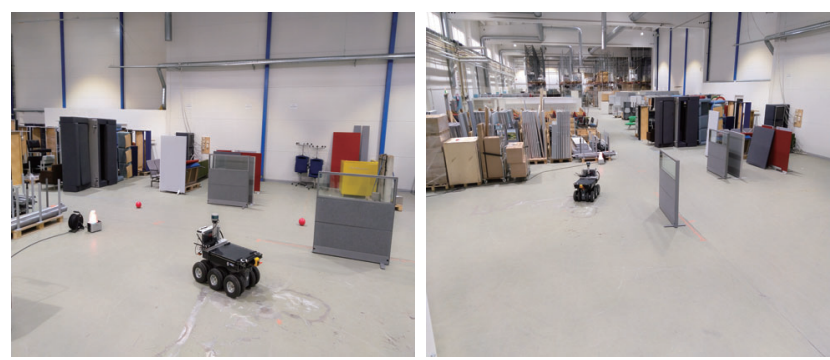

Fig. 7: Real world implementation of Mörri’s navigation

Some of the results from object detection and navigation visualization is shown in Figure 8 where 3D bounding boxes in different colors represent different classes. In the bottom image of Figure 8 which is showing two different object got detected in the same frame, in that you can see difference in cost map which doesn't show for soft ball, especially the small objects. This is the reason we showed marker visualization for object detection so that it reduces the collision. Finally, the overall results are provided and accomplished the task specific object detection and autonomous navigation in real-time has been achieved successfully and demonstrate with the Mörri UGV robot.
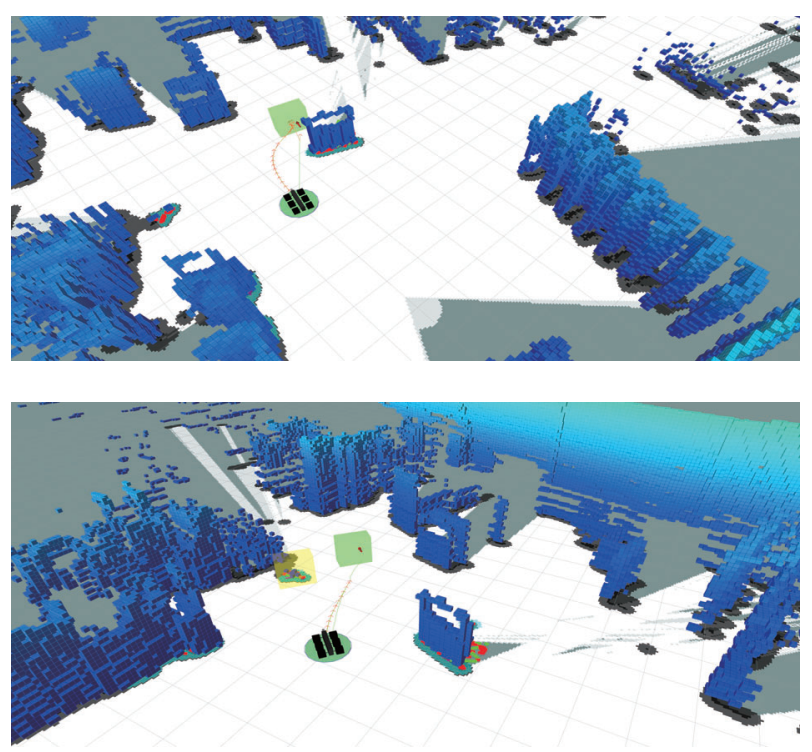

Fig. 8: Detected objects as markers in Rviz and TEB local planner solutions for target way-points

\section{CONCLUSION}

In this paper, we have successfully presented a working system for task-oriented autonomous UGV navigation with obstacle avoidance and object detection in a large warehouse. In future, overall brain node that contains the actions and plans for mapping the area autonomously and planning and scheduling other task should be implemented and also implementation of semantic segmentation to find the exact boundaries of the target.

\section{ACKNOWLEDGMENT}

We wish to thank University of Oulu which allowed us to collect the FSB Data-set in their warehouse. Our special thanks to Marko Kauppinen who helped with the data-set collection and providing technical support for Mörri.

\section{REFERENCES}

[1] Redmon, J., \& Farhadi, A. (2017). YOLO9000: better, faster, stronger. In Proceedings of the IEEE conference on computer vision and pattern recognition (pp. 7263-7271).

[2] Bashiri, F. S., LaRose, E., Peissig, P., \& Tafti, A. P. (2018). MCIndoor20000: A fully-labeled image data-set to advance indoor objects detection. Data in brief, 17, 71-75.

[3] Cordts, M., Omran, M., Ramos, S., Rehfeld, T., Enzweiler, M. Benenson, R., ... \& Schiele, B. (2016). The cityscapes data-set for semantic urban scene understanding. In Proceedings of the IEEE conference on computer vision and pattern recognition (pp. 32133223).

[4] Chen, S., Hong, J., Zhang, T., Li, J., \& Guan, Y. (2019, August). Object Detection Using Deep Learning: Single Shot Detector with a Refined Feature-fusion Structure. In 2019 IEEE International Conference on Real-time Computing and Robotics (RCAR) (pp. 219-224). IEEE.

[5] https://www.mbzirc.com/challenge/2020

[6] Kai O. Arras, José A. Castellanos, Martin Schilt, Roland Siegwart, "Feature-based multi-hypothesis localization and tracking using geometric constraints, Robotics and Autonomous Systems," Volume 44, Issue 1, 2003, Pages 41-53.

[7] JU, M., CHEN, Y., JIANG, W., Implementation of Odometry with EKF in Hector SLAM Methods. International Journal of Automation and Smart Technology, North America, 8, mar. 2018.

[8] S. Thrun, W. Burgard, and D. Fox, "Probabilistic Robotics." Cambridge, MA, USA: MIT Press, 2005.

[9] J. Folkesson and H. Christensen, "Graphical SLAM - a self-correcting map," IEEE International Conference on Robotics and Automation, 2004. Proceedings. ICRA '04. 2004, New Orleans, LA, USA, 2004, pp. 383-390 Vol.1.

[10] Kai M. Wurm, Cyrill Stachniss, Giorgio Grisetti, "Bridging the gap between feature- and grid-based SLAM, Robotics and Autonomous Systems," Volume 58, Issue 2, 2010, Pages 140-148.

[11] Stefan Kohlbrecher, Oskar von Stryk, Johannes Meyer, Uwe Klingauf. A flexible and scalable SLAM system with full 3D motion estimation[J]. 2011 IEEE International Symposium on Safety, Security, and Rescue Robotics,December 2011.

[12] A. Hornung, K. M. Wurm, M. Bennewitz, C. Stachniss, and W. Burgard, "OctoMap: an efficient probabilistic 3D mapping framework based on octrees," Auton. Robots, vol. 34, no. 3, pp. 189-206, Apr. 2013.

[13] C. Roesmann, W. Feiten, T. Woesch, F. Hoffmann and T. Bertram, "Trajectory modification considering dynamic constraints of autonomous robots," ROBOTIK 2012; 7th German Conference on Robotics, Munich, Germany, 2012, pp. 1-6.

[14] Moore T., Stouch D. (2016) A Generalized Extended Kalman Filter Implementation for the Robot Operating System. In: Menegatti E., Michael N., Berns K., Yamaguchi H. (eds) Intelligent Autonomous Systems 13. Advances in Intelligent Systems and Computing, vol 302. Springer, Cham.

[15] Fang, J., Zhou, L., \& Liu, G. (2019). 3D Bounding Box Estimation for Autonomous Vehicles by Cascaded Geometric Constraints and Depurated 2D Detections Using 3D Results. arXiv preprint arXiv:1909.01867. 